OPEN ACCESS

Edited by:

Francesco Visioli;

University of Padua, Italy

Reviewed by:

Ali Sadeghpour,

Tehran University of Medical

Sciences, Iran

Francesco Sofi,

Università degli Studi di Firenze, Italy

*Correspondence:

Jalal Moludi

jmoludi@yahoo.com

Specialty section:

This article was submitted to

Nutritional Epidemiology,

a section of the journal

Frontiers in Nutrition

Received: 12 September 2020

Accepted: 22 February 2021

Published: 06 April 2021

Citation:

Moludi J, Shivappa N

Alisgharzadeh S, Hébert JR and

Alizadeh M (2021) Dietary

Inflammatory Index Is Related to Heart

Failure Risk and Cardiac Function: A

Case-Control Study in Heart Failure

Patients. Front. Nutr. 8:605396.

doi: 10.3389/fnut.2021.605396

\section{Dietary Inflammatory Index Is Related to Heart Failure Risk and Cardiac Function: A Case-Control Study in Heart Failure Patients}

\author{
Jalal Moludi ${ }^{1,2 *}$, Nitin Shivappa ${ }^{3,4,5}$, Soghra Alisgharzadeh ${ }^{6}$, James R. Hébert ${ }^{3,4,5}$ and \\ Mohammad Alizadeh ${ }^{7}$ \\ ${ }^{1}$ School of Nutrition Sciences and Food Technology, Kermanshah University of Medical Sciences, Kermanshah, Iran, \\ ${ }^{2}$ Clinical Research Development Center, Imam Reza Hospital, Kermanshah University of Medical Sciences, Kermanshah, \\ Iran, ${ }^{3}$ Cancer Prevention and Control Program, University of South Carolina, Columbia, SC, United States, ${ }^{4}$ Department of \\ Epidemiology and Biostatistics, Arnold School of Public Health, University of South Carolina, Columbia, SC, United States, \\ ${ }^{5}$ Connecting Health Innovations LLC, Columbia, SC, United States, ${ }^{6}$ Student Research Committee, Tabriz University of \\ Medical Sciences, Tabriz, Iran, ${ }^{7}$ Nutrition Research Center, Faculty of Nutrition, Tabriz University of Medical Sciences, Tabriz, \\ Iran
}

Aims: Previous studies suggest that diet and inflammation are important risk factors for heart failure (HF); however, the associations remain unclear. The Dietary Inflammatory Index $\left(\mathrm{D} \|{ }^{\circledR}\right)$ was established to measure the inflammatory capacity of individuals' diet. This study aimed to explore the DII in HF subjects compared with controls.

Methods and Results: We conducted a case-control (116 cases and 113 controls) study that recruited in the similar clinics. DII scores were calculated based on dietary intakes. N-Terminal pro-brain natriuretic peptide (NT-proBNP) levels and ejection fraction (EF) were assessed in both groups. In order to analyze DIl scores with HF as the outcome, we used conditional logistic regression. A linear regression was applied to explore the associations between the DIl and left ventricular EF (LVEF).

There was statistically significant difference in DIl scores in cases vs. controls $(-0.16$ \pm 1.37 vs. $-0.33 \pm 1.67 ; p=0.040$ ). Conditional logistic regression has shown that subjects with higher DII scores had higher risk of HF. For every one-point rise in DII score, the odds of having HF increased by 30\% (OR: 1.30; Cl: 1.03, 1.69; $p=0.047$ ). The EF was inversely associated with saturated fatty acid $(\beta=-0.34,95 \% \mathrm{Cl}:-0.61,-0.07 ; p$ $=0.012)$. Subjects with higher DII scores had higher NT-proBNP levels and had lower EF.

Conclusion: The DII score was associated with high probability of HF. It appears that consumption of anti-inflammatory diet may lead to the prevention of HF and therefore suggests that dietary modification with the goal of reducing DII scores could be a valuable strategy for improving clinical outcomes in these patients.

Keywords: dietary inflammatory index, dietary intake, inflammation, case-control studies, heart failure 


\section{BACKGROUND}

Heart failure (HF) affects an estimated 23 million people worldwide (1) and leads to substantial numbers of hospitalizations and health-care costs. Therefore, prevention of HF has become a major public health concern, not just a major cause of its increasing prevalence but also because of its deleterious effect on quality of life (2). Low-grade inflammation is a principal factor leading to development of $\operatorname{HF}(3,4)$. Furthermore, erythrocyte sedimentation rate (ESR), as a marker of inflammation, was a significant predictor of $\operatorname{HF}(3,5)$. In particular, it has been shown that some environmental factors, such as dietary intake, are important in triggering an inflammatory response $(6,7)$.

The relationship between diet and HF is well-established. Healthy dietary patterns such as the Mediterranean diet are independently connected with a lower possibility of all-cause cardiovascular and cancer-related mortality $(8,9)$. Several studies have shown that certain nutrients can help alleviate diseases and modify systemic inflammation (9-12). On the other hand, diets with high sugar, refined starches, and saturated and transfatty acids and are poor in antioxidants and fibers may cause an activation of chronic inflammation (13). Undeniably, dietary patterns have long been known as potent factors leading to the acceleration of HF pathogenesis (14). Many dietary components have long been supposed to play a vital role in development of inflammation via both anti-inflammatory and pro-inflammatory mechanisms. Therefore, it is important to develop a scoring algorithm that takes into account these influences within an overall dietary pattern. Although most of the studies focus on whole diet in underlying mechanisms leading to HF development (15), knowledge about dietary components of that specific dietary components is still limited especially because the pathways leading to HF are not fully understood.

The dietary inflammatory index (DII $\left.{ }^{\circledR}\right)$ has been designed to quantify the potential inflammatory properties of a diet (16). The association between DII and various inflammatory markers has been established by multiple cohorts $(17,18)$. A large growing body of data investigating the associations between DII and risk of a wide range of non-communicable diseases including obesity, diabetes, cancers, and cardiovascular disease (CVD) has emerged (18-21).

The DII is not just restricted to macronutrients and micronutrients; it also includes flavonoids and frequently consumed parts of the diet such as tea and spices. The potential interactions among nutrients also must be taken into account (22). Moreover, individuals with greater DII had more risk of metabolic disorders $(19,23)$. As start and development of HF is related to a chronic pro-inflammatory state (24), we hypothesized that higher DII scores (or higher DII quartiles) are associated

\footnotetext{
Abbreviations: ANOVA, analysis of variance; CAD, coronary artery disease; CRP, C-reactive protein; CVD, cardiovascular disease; NT-proBNP, N-terminal (NT)pro hormone BNP; LVEF, left ventricular ejection fraction; ESR, erythrocyte sedimentation rate; DII, Dietary Inflammatory Index; HF, heart failure; HDL, high-density lipoprotein cholesterol; LDL, low-density lipoprotein cholesterol; NYHA, New York Heart Association; TC, total cholesterol; TNF- $\alpha$, tumor necrosis factor- $\alpha$.
}

with $\mathrm{HF}$ and worse clinical outcomes. Based on previous research with the DII and evidence linking inflammation with CVD, this study aimed to examine the relationship between the DII and HF in a case-control research conducted in Iran.

\section{METHODS}

\section{Subjects}

The current hospital-based matched case-control study was conducted from January 2017 through February 2018 in cardiac medical centers in Tabriz, East Azerbaijan Province, Iran. Subjects with HF were recruited by convenience sampling from the Madani Heart Center under the supervision of Tabriz University of Medical Sciences. The hospital is a single specialty heart hospital, which is the largest of its kind in the northwest of the country, with 330 administrative and clinical staff.

The sample size calculation has been explained before. Briefly, the sample size was designed by the following formula: $n=$ $\left[(\mathrm{Z} \alpha / 2+\mathrm{Z} \beta) 2 \times\{(\mathrm{p} 1(1-\mathrm{p} 1)+(\mathrm{p} 2(1-\mathrm{p} 2))\}] /(\mathrm{p} 1-\mathrm{p} 2)^{2}\right.$, where $\mathrm{p} 1$ is the proportion of the subjects with low DII, $\alpha$-error $=0.05$, and power $=80 \%(1-\beta)(25)$. Consequently, a sample size of 120 patients was calculated for the study (120 in each group). We also assumed $5 \%$ loss $(125+5)$, and the final sample size of 250 was included in this study.

Patients with HF clinically defined based on the European Society of Cardiology guidelines, who were established less than a year before enrolment, were involved in the study. Other inclusion criteria were subjects aged 30-70 years and did not announce modifications to their diets from the time during diagnosis. We included only new cases in the study with the intention of lessening the possibility that subjects altered their diet considerably in during diagnosis. Patients were diagnosed to have HF if they had had left ventricular ejection fraction (LVEF) $\leq 0.35$, were in New York Heart Association (NYHA) functional classification class II to IV HF for $\geq 3$ months before study, had a heart rate $\geq 68 \mathrm{bpm}$, and were receiving standard therapy of diuretics and an angiotensin-converting enzyme (ACE) inhibitor or digoxin. HF cases were patients who had two-dimensional echocardiography and confirmed by clinical symptoms. Also, the patients with preserved EF but with clinical symptoms were also considered in this study. Exclusion criteria in the case group included the following: (a) non-adherence to the study protocol, (b) reporting caloric intake $>4,000$ or $<800 \mathrm{kcal} / \mathrm{day}$, (c) inability to respond to the questions, and (d) supplement therapy for HF.

Controls $(n=125)$ were randomly selected from the same hospital who were admitted to the hospitals and underwent percutaneous coronary intervention (PCI) and no evidence of cardiomyopathy $(\mathrm{EF} \geq 40)$ but who might have hyperlipidemia and hypertension $(n=125)$. Exclusion criteria for controls were prior diagnosis of inflammatory diseases of the peripheral nervous system; cancer; liver diseases and gastrointestinal, metabolic, and endocrine disorders; and immune system disorders and have a special diet (e.g., vegetarian or weightloss diet) and low cardiac function $(\mathrm{EF}<40)$. Patients with acute myocardial infarction (MI) and decreased cardiac function were excluded. Controls were coordinated to cases on age ( \pm 4 years) and sex. All of the patients were screened by an 


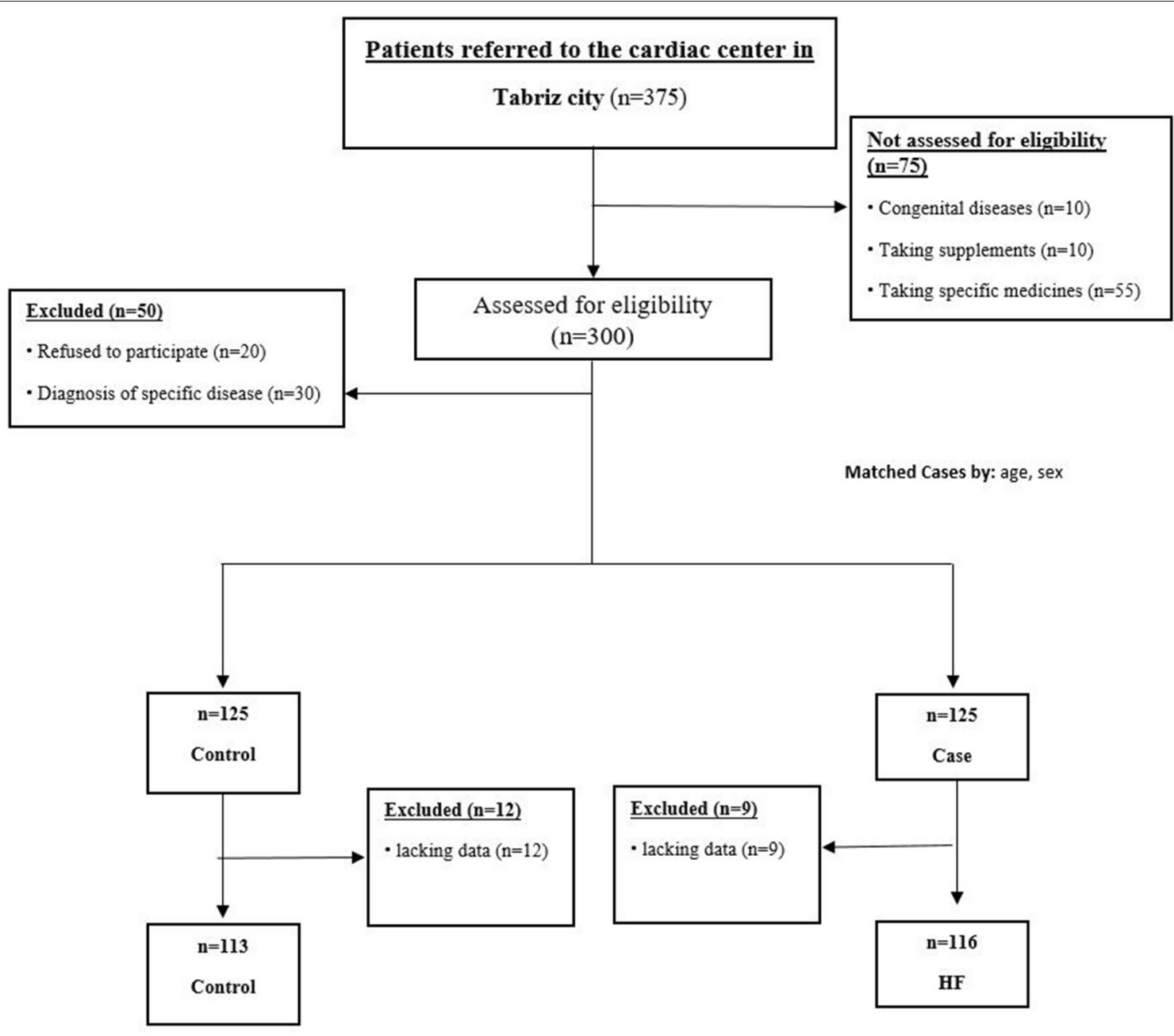

FIGURE 1 | Flowchart of study.

expert cardiologist for eligibility. Of joined cases and controls, nine $\mathrm{HF}$ and 12 controls were excluded because of lacking food frequency questionnaires (FFQs) or other data. The final sample comprised 116 cases and 113 controls, demonstrating a 90\% response rate (Figure 1). Informed consent was obtained from all subjects. The research was approved by the research council of the Tabriz University of Medical Sciences (No. IR.TBZMED.REC.1397.184). Information on sociodemographic characteristics, anthropometric data, biochemical variables, and medical history was assessed using a standard questionnaire by a trained interviewer.

\section{Assessment of Anthropometric Data}

We used a scale (Seca 770; Seca GmbH \& Co, Hamburg, Germany) with $0.5-\mathrm{kg}$ accuracy while patients were wearing light clothing and no shoes in order to measure the subjects' body weight. We also utilized a tape with $0.5-\mathrm{cm}$ accuracy in order to assess their height. We computed the body mass index (BMI) by weight-to-height ratio $\left(\mathrm{BMI}=\mathrm{kg} / \mathrm{m}^{2}\right)$.
$\mathrm{N}$-Terminal pro-brain natriuretic peptide (NT-proBNP) levels was assessed by the quantitative electrochemiluminescence immunoassay (ELISA) method (Roche kite).

\section{Determination of Ejection Fraction}

EF was measured by two-dimensional echocardiography. The participants were divided into EFs $\geq 40 \%$ (controls) and $<35 \%$ (HF case) subgroups. Furthermore, we used clinical symptoms, which confirmed classification.

\section{Dietary Assessment}

We obtained dietary intake by making use of a 138-item semi-quantitative FFQ involving of a list of a finite foods and beverages with standard serving sizes usually consumed by Iranians $(26,27)$. Patients were requested to state how often they consumed each of the food listed by the number of times (every day, weekly, once a month, and annually). According to a standard serving size for each nutrient item, and then each contributor's intake was transformed to weight equivalents (i.e., $\mu \mathrm{g}$, g, and $\mathrm{mg}$ ) per day. Then, dietary 
information was investigated for energy and nutrients, using revised Nutritionist IV software (Nutritional Database Manager 4.0.1, First Data Bank, USA). The development and validation of the DII are described in detail elsewhere (22). The greater the DII score, the more pro-inflammatory the diet. More negative values represent the more anti-inflammatory diets. The DII tertile cut points were categorized based on cut points: Tertile $1 \leq-1.132$; Tertile $2=-1.132$ to 0.467 ; Tertile $3 \geq 0.467$.

TABLE 1 | Characteristics of patients in the case and control groups $(n=229)$.

\begin{tabular}{|c|c|c|c|}
\hline Characteristics & Cases $(n=116)$ & Controls $(n=113)$ & $p$-value \\
\hline Age, years & $56.34 \pm 12.99$ & $57.40 \pm 13.43$ & 0.547 \\
\hline \multicolumn{4}{|l|}{ Gender, n (\%) } \\
\hline Male & 75 (64.66) & $69(61.06)$ & 0.573 \\
\hline Female & $41(35.34)$ & $44(38.94)$ & \\
\hline $\mathrm{BMI}, \mathrm{kg} / \mathrm{m}^{2}$, mean $\pm \mathrm{SD}$ & $26.63 \pm 5.75$ & $26.73 \pm 5.29$ & 0.892 \\
\hline \multicolumn{4}{|l|}{ Smoking, n (\%) } \\
\hline Yes & $35(30.17)$ & $22(19.47)$ & 0.061 \\
\hline No & $81(69.83)$ & $91(80.53)$ & \\
\hline \multicolumn{4}{|l|}{ Hypertension, n (\%) } \\
\hline Yes & $61(52.59)$ & $51(45.13)$ & 0.259 \\
\hline No & $55(47.41)$ & $62(54.87)$ & \\
\hline EF (\%) & $25.34 \pm 9.70$ & $43.3 \pm 19.5$ & 0.001 \\
\hline $\mathrm{LDL}, \mathrm{mg} / \mathrm{dl}$, mean $\pm \mathrm{SD}$ & $131.60 \pm 30.14$ & $120.37 \pm 34.84$ & 0.010 \\
\hline HDL mg/dl, mean \pm SD & $39.47 \pm 7.26$ & $44.81 \pm 9.15$ & 0.000 \\
\hline Cholesterol mg/dl, mean \pm SD & $180.89 \pm 47.24$ & $173.11 \pm 46.43$ & 0.210 \\
\hline $\mathrm{TG}, \mathrm{mg} / \mathrm{dl}$, mean $\pm \mathrm{SD}$ & $151.34 \pm 48.62$ & $139.90 \pm 48.26$ & 0.075 \\
\hline Dll, mean \pm SD & $-0.16 \pm 1.37$ & $-0.33 \pm 1.67$ & 0.040 \\
\hline
\end{tabular}

Student $t$-test was used for continuous variables. Chi-square test was used for categorical variables. Significance was at $p<0.05$. $p$-values are based on Mann-Whitney U-test. DII, dietary inflammatory index; BMI, body mass index; EF, ejection fraction; $L D L$, lowdensity lipoprotein; HDL, high-density lipoprotein; TG, triglycerides.

\section{Data Analysis}

The data were examined by SPSS 16 software (SPSS Inc., Chicago, IL, USA). Data are presented as mean and standard deviation, frequency and percent, or median and interquartile range. The normality of data was assessed and proved by skewness and kurtosis test. For all statistical assessments, $p<0.05$ was considered as significant. Independent samples $t$-test and MannWhitney $U$-test were used for between-group differences. $p$ values for trends were calculated for variables across DII tertiles. Conditional logistic regression was done to calculate odds ratio (OR) and 95\% confidence intervals (CIs) for HF as the outcome. Multiple linear regressions via the enter method were used to evaluate the associations between EF as outcome and continuous outcomes such as DII, sex, age, and BMI.

\section{RESULTS}

Table 1 indicates the distribution of $\mathrm{HF}$ and controls with reference to some variables. DII scores in our study ranged from -3.3 (maximum anti-inflammatory score) to +2.82 (most proinflammatory score). By design, the demographic distributions were not different in cases and controls. Furthermore, the control group had higher values of high-density lipoprotein (HDL) ( $p=$ 0.001 ) and lower level of low-density lipoprotein (LDL) than the case groups $(p=0.01)$.

The results also indicated that the mean DII score for the HF group was higher than that of the control group $(-0.16 \pm 1.37$ vs. $-0.33 \pm 1.67 ; p=0.040)$. When DII scores were transformed toward tertiles (Table 2), cumulative trends across tertiles of DII were detected for total fat and saturated fat, whereas statistically substantial decreasing trends were perceived for some anti-inflammatory intake such as fiber and beta carotene. However, in the analysis via the DII shown as tertiles, we failed to find any significant trend of increasing risk $(p>0.05)$ for other cardiovascular risk

TABLE 2 | Characteristics of the participants according to the quartiles of dietary inflammatory index.

\begin{tabular}{|c|c|c|c|c|}
\hline Variables & Tertile 1 & Tertile 2 & Tertile 3 & $p_{\text {trend }}$ \\
\hline \multicolumn{5}{|l|}{ Demographic } \\
\hline Age (years) & $56.79 \pm 10.44$ & $56.89 \pm 14.90$ & $56.98 \pm 14.13$ & 0.995 \\
\hline Gender, n (\%) & & & & 0.735 \\
\hline Male & $46(61.33)$ & $48(63.16)$ & $48(64.00)$ & \\
\hline $\mathrm{BMI}(\mathrm{kg} / \mathrm{m})$ & $26.43 \pm 4.71$ & $27.09 \pm 6.38$ & $26.61 \pm 5.42$ & 0.752 \\
\hline Smoking, n (\%) & $24(32.00)$ & $17(22.37)$ & $15(20.00)$ & 0.090 \\
\hline \multicolumn{5}{|l|}{ Pro-inflammatory } \\
\hline Total fat (g) & $71.05 \pm 30.13$ & $84.75 \pm 41.19$ & $94.23 \pm 44.92$ & $<0.001$ \\
\hline Cholesterol (mg) & $231.44 \pm 155.77$ & $202.39 \pm 98.96$ & $194.97 \pm 108.23$ & 0.198 \\
\hline Saturated fat (g) & $19.00(9.00,23.19)$ & $17.39(9.74,20.53)$ & $30.70(18.53,33.41)$ & $<0.001$ \\
\hline \multicolumn{5}{|l|}{ Anti-inflammatory } \\
\hline Fiber (g) & $18.41 \pm 8.82$ & $4.00 \pm 1.44$ & $13.35 \pm 5.73$ & $<0.001$ \\
\hline Beta carotene $(\mu \mathrm{g})$ & $267.17(97.72,451.10)$ & $73.16(23.49,519.60)$ & $146.00(17.51,296.66)$ & $<0.001$ \\
\hline
\end{tabular}

For abbreviations, see Table 1. Values are presented as mean $\pm S D$ for variables with normal distribution or median (interquartile) for variables without normal distribution. Tertile $1 \leq-1.132 ;$ Tertile $2=-1.132$ to $0.467 ;$ Tertile $3 \geq 0.467$ 
TABLE 3 | Micronutrient intake of the participants according to the tertiles of dietary inflammatory index.

\begin{tabular}{|c|c|c|c|c|}
\hline Variables & Tertile 1 & Tertile 2 & Tertile 3 & $p_{\text {trend }}$ \\
\hline Sodium (mg) & $1115.00(775.10,2038.00)$ & $1119.50(499.00,1973.00)$ & 1999.00 (1367.00, 2131.00 & 0.020 \\
\hline Iron (mg) & $14.51(1,232,14.94)$ & $13.11(9.20,16.95)$ & $12.87(11.80,17.88)$ & 0.147 \\
\hline Zinc (mg) & $7.91(5.81,10.75)$ & $7.37(5.17,10.54)$ & $9.15(7.31,1,032)$ & 0.513 \\
\hline Magnesium (mg) & $254.7(181.27,272.43)$ & $195.50(151.60,232.70)$ & $202.87(183.70,213.50)$ & 0.006 \\
\hline Manganese (mg) & $2.18(1.88,2.46)$ & $1.93(1.39,2.48)$ & $1.60(1.26,2.11)$ & 0.401 \\
\hline Potassium (mg) & $2728.00(2375.00,3636.00)$ & $2067.00(1696.50,2466.67)$ & $2588.5(2318.00,2676.00)$ & 0.044 \\
\hline Calcium (mg) & $694.67(509.33,1054.00)$ & $663.30(389.67,817.50)$ & $679.00(526.30,1077.00)$ & 0.969 \\
\hline Phosphorus (mg) & $1012.00(785.40,1255.00)$ & $989.65(611.87,1,113.00)$ & $1109.00(1055.00,1148.00)$ & 0.589 \\
\hline Copper & $1.34(1.09,1.47)$ & $0.99(0.77,1.46)$ & $1.09(0.87,1.22)$ & 0.005 \\
\hline Selenium & $0.10(0.09,0.14)$ & $0.11(0.08,0.15)$ & $0.08(0.05,0.17)$ & 0.048 \\
\hline Chromium & $0.05(0.03,0.08)$ & $0.04(0.02,0.06)$ & $0.05(0.02,0.07)$ & 0.209 \\
\hline Vitamin A & $802.67(686.10,922.70)$ & $416.00(271.80,843.80)$ & $422.30(352.5,502.33)$ & $<0.001$ \\
\hline Vitamin E & $4.79(2.98,7.24)$ & $3.57(2.09,5.56)$ & $3.57(2.51,6.08)$ & 0.031 \\
\hline Vitamin K & $70.28(44.90,114.40)$ & $30.67(18.29,50.66)$ & $40.48(19.05,74.33)$ & $<0.001$ \\
\hline Vitamin D & $1.00(0.21,1.99)$ & $1.05(0.04,2.00)$ & $0.79(0.03,2.11)$ & 0.634 \\
\hline Thiamin & $2.28(1.65,2.46)$ & $1.63(1.31,2.07)$ & $1.60(1.48,1.92)$ & $<0.001$ \\
\hline Riboflavin & $1.56(1.11,1.92)$ & $1.42(0.87,1.56)$ & $1.52(1.48,1.83)$ & 0.268 \\
\hline Niacin & $19.88(17.21,24.98)$ & $16.78(12.83,21.00)$ & $17.79(14.34,22.89)$ & 0.060 \\
\hline Pantothenic acid & $4.92(4.09,5.64)$ & $3.83(2.52,4.81)$ & $3.94(3.83,4.57)$ & $<0.001$ \\
\hline Pyridoxine & $1.38(1.08,2.27)$ & $0.98(0.80,1.44)$ & $1.11(0.93,1.45)$ & $<0.001$ \\
\hline Folate & $210.40(186.13,251.60)$ & $135.00(101.28,245.20)$ & $155.20(131.67,199.20)$ & $<0.001$ \\
\hline Cobalamin & $2.83(2.31,3.34)$ & $2.83(1.66,3.70)$ & $3.51(1.98,4.08)$ & 0.474 \\
\hline Biotin & $23.94(17.11,26.48)$ & $15.37(10.98,21.95)$ & $16.84(11.52,21.48)$ & $<0.001$ \\
\hline
\end{tabular}

Data are median (interquartile range).

Tertile $1 \leq-1.132$; Tertile $2=-1.132$ to 0.467 ; Tertile $3 \geq 0.467$.

factors including lipid profile. Also, when DII was transformed into tertiles according to DII, there are some differences observed between the micronutrient intake of the participants (Table 3).

Clinical markers by DII tertiles are presented in Table 4. Especially, members in tertiles 3 had higher NT-proBNP and had lower EF (Table 4). The present results led us to conclude that low DII score leads to better clinical outcome in HF patients. However, in the analysis via the DII shown as tertiles, we failed to find any significant trend $(p>0.05)$ for other cardiovascular risk factors including lipid profile.

Logistic regression analysis was done with $\mathrm{HF}$ as the outcome (Table 5), and the findings showed that each unit increase in DII score was associated with a $30 \%$ (OR: 1.30; CI: 1.03, 1.69; $p=0.047$ ) increase in the odds of being diagnosed with HF. Significant OR also was perceived for triglyceride (TG), LDL, and HDL.

In a linear regression model including LVEF as the outcome, EF value was inversely associated with saturated fatty acid ( $\beta$ $=-0.34,95 \%$ CI: $-0.61,-0.07 ; p=0.012$ ) (Table 6). No statistically significant relationship was established between the DII and the LVEF value. Finally, the set of variables including DII, HDL, TG, LDL, and saturated fatty acid significantly explained $9 \%$ of variance in $\mathrm{EF}$ status $\left[F_{(9,215)}=3.54, p \leq 0.001\right]$.
TABLE 4 | Distribution of some important clinical markers across categories of DII.

\begin{tabular}{lcccc}
\hline Variables & Tertile 1 & Tertile 2 & Tertile 3 & $\boldsymbol{p}_{\text {trend }}$ \\
\hline EF & $43.12 \pm 24.94$ & $37.08 \pm 14.23$ & $33.11 \pm 12.76$ & $\mathbf{0 . 0 4 4}$ \\
LDL $(\mathrm{mg} / \mathrm{dll})$ & $123.05 \pm 31.68$ & $128.03 \pm 33.62$ & $126.96 \pm 34.36$ & 0.627 \\
HDL $(\mathrm{mg} / \mathrm{dll})$ & $42.07 \pm 9.22$ & $43.00 \pm 8.50$ & $41.37 \pm 8.35$ & 0.515 \\
Cholesterol $(\mathrm{mg} / \mathrm{dl})$ & $174.64 \pm 41.75$ & $169.46 \pm 46.93$ & $187.80 \pm 50.11$ & 0.371 \\
TG (mg/dl) & $145.81 \pm 52.24$ & $149.43 \pm 44.22$ & $141.17 \pm 49.83$ & 0.582 \\
NT-proBNP & $109.81 \pm 47.24$ & $101.53 \pm 43.12$ & $155.17 \pm 59.21$ & $\mathbf{0 . 0 3 6}$
\end{tabular}

For abbreviations, see Table 1. Values are presented as mean $\pm S D$ for variables with normal distribution or median (interquartile) for variables without normal distribution. Tertile $1 \leq-1.132$; Tertile $2=-1.132$ to 0.467 ; Tertile $3 \geq 0.467$.

Bold values are statistically significant.

\section{DISCUSSION}

The current case-control study applied the DII score to the dietary intake of patients with HF for the first time. We detected that the DII score was related to increased risk of HF, thus implicating a pro-inflammatory diet in its etiology. However, EF was not associated with DII score.

It has been claimed that inflammation has a significant impact on the pathogenesis of CVDs and that higher highsensitivity C-reactive protein (CRP) concentration accelerates 
TABLE 5 | Conditional logistic regression analysis with HF status as outcome.

\begin{tabular}{lccr}
\hline Variables & Odds ratio & $\mathbf{9 5 \%} \mathbf{C l}$ & $\boldsymbol{p}$-value \\
\hline DIl & 1.303 & $1.003,1.693$ & 0.047 \\
HDL & 1.127 & $1.060,1.198$ & $<0.001$ \\
TG & 0.988 & $0.979,0.997$ & 0.008 \\
LDL & 0.985 & $0.972,0.998$ & 0.034 \\
Saturated fatty acids & 0.905 & $0.861,0.951$ & $<0.001$ \\
\hline
\end{tabular}

For abbreviations, see Table 1.

Adjusted for BMI and smoking.

TABLE 6 | Summary of linear regression analysis based on EF as outcome.

\begin{tabular}{lcccc}
\hline Variables & B & SE & $\mathbf{9 5 \%} \mathbf{C l}$ & $\boldsymbol{p}$-value \\
\hline DII & 0.73 & 0.85 & $-1.12,-0.03$ & 0.389 \\
HDL & 0.28 & 0.13 & $0.01,0.55$ & 0.038 \\
TG & -0.08 & 0.02 & $-0.12,-0.03$ & 0.001 \\
LDL & -0.06 & 0.03 & $-0.13,0.01$ & 0.090 \\
Saturated fatty asides & -0.34 & 0.13 & $-0.61,-0.07$ & 0.012 \\
Adjusted $R^{2}$ & 0.09 & & & \\
\hline
\end{tabular}

For abbreviations, see Table 1. Adjusted for age, sex, BMI, and smoking.

the development of HF (28). Similarly, plasma levels of tumor necrosis factor- $\alpha$ (TNF- $\alpha)$ and IL- 6 also predict HF outcomes (29). In addition, as beginning and development of HF accompany the chronic pro-inflammatory state, extra proinflammatory dietary patterns are associated with increased $\mathrm{HF}$ incidence and worse clinical outcome (30). One potential mechanism for the apparent relationship between the DII score and high risk of inflammatory diseases like CVD is the impact of diet on the cytokines levels, which regulate inflammatory response. Inflammatory cytokines also play a vital role in the underlying pathophysiological processes of HF (31).

While there are many studies supporting the concept of the protective effect of diets, including approaches to stop hypertension [Paleolithic, Dietary Approaches to Stop Hypertension (DASH) diet, low carb diet, vegetarian diet, and low-fat diet] on the prevention of HF, their effectiveness is not clear yet. DASH and Mediterranean diets revealed a protective impact on the HF incidence and/or deteriorating on $\operatorname{EF}(13,32)$, but these results need to be able to replicate and investigate other diets (such as low DII) and to test the generalizability in post-MI patients. In other words, the consumption of vegetables and fruits has been proven to decrease inflammation, whereas the consumption of foods like meat and butter increases inflammation through increasing levels of CRP $(33,34)$. Indeed, focusing on the entire diet by computing DII score, which takes many categories of dietary components into account, more precisely indicates the relationship between diet and the risk of $\mathrm{HF}$.

Previous findings have shown the relationship between the DII score and incidence of CVD (MI, stroke, and CVD death) (19). Numerous studies have indicated the significant role of chronic inflammation as an important factor that interferes in the development of CVD. In this context, dietary patterns are adjustable factors that have a huge potential to exert a powerful anti-inflammatory or pro-inflammatory effect (35). We observed a higher DII score in HF subjects compared with controls. This result is consistent with a previous systematic review of studies, which found that the DII scores were inversely associated with cardio-metabolic risk factors and CVDs (36). Therefore, a diet including pro-inflammatory components such as saturated and trans fat can cause proliferation of MI, oxidative stress $(37,38)$, and inflammation, which can cause ventricular remodeling and possibly HF development.

Our finding is consistent with previous result for DII and chronic disease in many clinical settings. Previously, we observed a negative association between DII and EF, which agrees with a previous SUN ("Seguimiento University of Navarra") cohort study that was conducted in 18,794 individuals with 8.9 years' follow-up, where the number of new CVD events cases in the highest DII score was 2.03 times more than the lowest quartile (39). In another cohort study, carried out on 7,216 subject (5580 years) at high risk of cardiovascular events, the highest quartile (most pro-inflammatory diet) was reversely associated with the number of CVD incidences (OR $=1.73 ; 95 \%$ CI: 1.15, 2.60) (40). A comparable negative relationship was reported between HF risk and DII score in patients with previously diagnosed CVDs $(\mathrm{OR}=0.31 ; 95 \% \mathrm{CI}: 0.12,0.82 ; p=0.018)$ (41). In contrast, the SU.VI.MAX study included 7,743 participants (aged 35-60 years) after 11.4 years' follow-up; no associations were observed for DII scores and the composite CVD outcomes (32). Effect sizes for $\mathrm{HF}$ risk have mostly been in the range of nearly OR 1.5-3, which is similar to our results. By comparing the results from this study, we hope to determine the association between DII and HF. The DII scores also in current population were comparable with those of earlier studies. The DII based on FFQ in our study population seems to be acceptable for this type of examination and for interpretation of the results in relation to earlier results. The DII score was highly positively associated with the intakes of saturated fatty acid. The higher intakes of saturated fatty acid are associated with inflammatory properties $(5,31)$; so it is not unexpected that there was a correlation with $\mathrm{HF}$ as reported by DII.

Numerous investigations have revealed a positive correlation between lipid profile (including total cholesterol, LDL, and HDL) and risk of HF and clinical outcomes $(42,43)$. The lack of a significant association in our study may be partially a result of the low levels of these outcomes among participants at baseline; also $60 \%$ of patients were under statin therapy. Low serum total cholesterol is related with increased mortality in patients with HF (43). In particular, HDL cholesterol was proposed to exert anti-inflammatory and antioxidant activities, which would lessen the pro-inflammatory state in patients with HF (44). Indeed, an association among HDL cholesterol, HF, and DII may be explained on the basis of the inflammation hypothesis (24), which suggests that low DII score modulates inflammatory immune function and also that HDL cholesterol has antioxidant and antiinflammatory properties, both of which can further reduce risk of HF. 
The potential cardiovascular effects of several foods and dietary patterns are still incompletely understood. Some research has suggested that "Western" dietary patterns, which contain high intakes of saturated and trans fatty acids, and low-fruit/lowvegetable intake, result in higher CRP levels and increased risk of CVD event $(5,10,31,40)$. In this regard, it seems that the dietary patterns with low DII scores prevent the development of HF.

Previously, the DII has been revealed to be related with different inflammatory markers including homocysteine and CRP (9). Diets having high amounts of vegetables and fruits are related with low levels of hs-CRP (34). Certain nutrients such as magnesium, fiber, and omega-3 fatty acids are associated with low levels of inflammation (14). A further novel finding is that those higher in DII had higher NT-proBNP and had lower EF, which mean low intensity of HF. The NT-proBNP levels were measured in all patients, and they were followed up and had a well-accepted biomarker of cardiac function (45). The present work led us to conclude that low DII score is associated with better cardiac function in HF patients.

This is the first study in the world to explore the relationship between the DII score and HF. Despite its strengths, our study had several limitations. First, we did not quantity inflammatory biomarkers, and as a result, we were incapable of evaluating this association. The second limitations relate to the generalizability of our results because of its design, which does not permit the distinction between cause and effect based on temporality. Therefore, we were unable to determine if a pro-inflammatory dietary pattern and higher DII score cause HF development or whether the existence of $\mathrm{HF}$ resulted in a more proinflammatory diet, which in turn resulted in higher DII. Thus, perspective studies, including intervention trials, are required to determine the role of dietary intakes as a cause of HF more firmly.

\section{CONCLUSION}

In summary, the DII has presented a valuable means for evaluating the potential inflammatory of diets in HF patients. This study on HF patients conducted in Iranian subjects indicated a possible role of diet through the development of inflammation. Our results proposed evidence suggesting that

\section{REFERENCES}

1. Khatibzadeh S, Farzadfar F, Oliver J, Ezzati M, Moran A. Moran worldwide risk factors for heart failure: a systematic review and pooled analysis. Int $J$ Cardiol. (2013) 168:1186-94. doi: 10.1016/j.ijcard.2012.11.065

2. Ambrosy AP, Fonarow GC, Butler J, Chioncel O, Greene SJ, Vaduganathan $\mathrm{M}$, et al. The global health and economic burden of hospitalizations for heart failure: lessons learned from hospitalized heart failure registries. J Am College Cardiol. (2014) 63:1123-33. doi: 10.1016/j.jacc.2013.11.053

3. Ingelsson E, Arnlöv J, Sundström J, Lind L. Inflammation, as measured by the erythrocyte sedimentation rate, is an independent predictor for the development of heart failure. J Am College Cardiol. (2005) 45:1802-6. doi: 10.1016/j.jacc.2005.02.066

4. Kalogeropoulos A, Georgiopoulou V, Psaty BM, Rodondi N, Smith AL, Harrison DG, et al. Inflammatory markers and incident heart failure risk in an upper DII score (representative an extra pro-inflammatory diet) is strictly connected with the occurrence of HF. These results propose the significance of promoting dietary intake with low DII score for the subjects at risk for HF. Future studies, including intervention trials, are required to find this association precisely. The significance of this work is of major importance for public health-care planners, since it would test the low inflammatory diet hypothesis at population basis and may provide an additional, non-pharmacologic means for the prevention of HF.

\section{DATA AVAILABILITY STATEMENT}

The raw data supporting the conclusions of this article will be made available by the authors, without undue reservation.

\section{ETHICS STATEMENT}

The studies involving human participants were reviewed and approved by the research council of the Tabriz University of Medical Sciences (No: IR.TBZMED.REC.1397.184). The patients/participants provided their written informed consent to participate in this study.

\section{AUTHOR CONTRIBUTIONS}

JM and MA: conceived and designed the experiments. JM performed the experiments. SA, JM, and NS: analyzed the data. All authors: wrote the paper.

\section{FUNDING}

This study was supported financially by TBZMED as a thesis proposal for the Ph.D. degree of the JM. The funders of the study had no role in study design, data collection, data analysis, data interpretation, or writing of the report.

\section{ACKNOWLEDGMENTS}

We would like to thank all contributors of the current study for their concepts, ideas, contribution, and provision. 
disease and all-cause mortality. Cancer Epidemiol Prevent Biomark. (2011) 20:1089-97. doi: 10.1158/1055-9965.EPI-10-1173

9. Catapano G, Pedone C, Nunziata E, Zizzo A, Passantino A, Incalzi RA. Nutrient intake and serum cytokine pattern in elderly people with heart failure. Eur J Heart Failure. (2008) 10:428-34. doi: 10.1016/j.ejheart.2008.02.016

10. Ciccone MM, Cortese F, Gesualdo M, Carbonara S, Zito A, Ricci G, et al. Dietary intake of carotenoids and their antioxidant and anti-inflammatory effects in cardiovascular care. Med Inflamm. (2013) 2013:782137. doi: $10.1155 / 2013 / 782137$

11. Sinatra ST. Coenzyme Q10: a vital therapeutic nutrient for the heart with special application in congestive heart failure. Connecticut Med. (1997) 61:707-12.

12. Schroten NF, Ruifrok WP, Kleijn L, Dokter MM, Silljé HH, Lambers Heerspink HJ, et al. Short-term vitamin D3 supplementation lowers plasma renin activity in patients with stable chronic heart failure: an open-label, blinded end point, randomized prospective trial (VitDCHF trial). Am Heart J. (2013) 166:357-64.e2. doi: 10.1016/j.ahj.2013. 05.009

13. Nettleton JA, Steffen LM, Mayer-Davis EJ, Jenny NS, Jiang R, Herrington $\mathrm{DM}$, et al. Dietary patterns are associated with biochemical markers of inflammation and endothelial activation in the Multi-Ethnic Study of Atherosclerosis (MESA)-. Am J Clin Nutr. (2006) 83:1369-79. doi: $10.1093 /$ ajcn/83.6.1369

14. He J, Ogden LG, Bazzano LA, Vupputuri S, Loria C, Whelton PK. Dietary sodium intake and incidence of congestive heart failure in overweight US men and women: first national health and nutrition examination survey epidemiologic follow-up study. Arch Internal Med. (2002) 162:1619-24. doi: 10.1001/archinte.162. 14.1619

15. Levitan EB, Wolk A, Mittleman MA. Consistency with the DASH diet and incidence of heart failure. Arch Internal Med. (2009) 169:851-7. doi: 10.1001/archinternmed.2009.56

16. Gambardella J, Santulli G. Integrating diet and inflammation to calculate cardiovascular risk. Atherosclerosis. (2016) 253:258. doi: 10.1016/j.atherosclerosis.2016.08.041

17. Shivappa N, Steck SE, Hurley TG, Hussey JR, Ma Y, Ockene IS, et al. A population-based dietary inflammatory index predicts levels of C-reactive protein in the Seasonal Variation of Blood Cholesterol Study (SEASONS). Public Health Nutr. (2014) 17:1825-33. doi: 10.1017/S13689800130 02565

18. Shivappa N, Hébert JR, Rietzschel ER, De Buyzere ML, Langlois M, Debruyne E, et al. Associations between dietary inflammatory index and inflammatory markers in the Asklepios study. Brit J Nutr. (2015) 113:665-71. doi: 10.1017/S000711451400395X

19. Garcia-Arellano A, Ramallal R, Ruiz-Canela M, Salas-Salvadó J, Corella D, Shivappa N, et al. Dietary inflammatory index and incidence of cardiovascular disease in the PREDIMED study. Nutrients. (2015) 7:4124-38. doi: 10.3390/nu7064124

20. Phillips CM, Shivappa N, Hébert JR, Perry IJ. Dietary inflammatory index and biomarkers of lipoprotein metabolism, inflammation and glucose homeostasis in adults. Nutrients. (2018) 10:1033. doi: 10.3390/nu100 81033

21. Shivappa N, Bosetti C, Zucchetto A, Montella M, Serraino D, La Vecchia C, et al. Association between dietary inflammatory index and prostate cancer among Italian men. Brit J Nutr. (2015) 113:278-83. doi: $10.1017 /$ S0007114514003572

22. Shivappa N, Steck SE, Hurley TG, Hussey JR, Hébert JR. Designing and developing a literature-derived, population-based dietary inflammatory index. Public health Nutr. (2014) 17:1689-96. doi: 10.1017/S1368980013002115

23. Ahluwalia N, Andreeva VA, Kesse-Guyot E, Hercberg S. Dietary patterns, inflammation and the metabolic syndrome. Diabetes Metabo. (2013) 39:99110. doi: 10.1016/j.diabet.2012.08.007

24. Dick SA, Epelman S. Chronic heart failure and inflammation: what do we really know? Circulat Res. (2016) 119:159-76. doi: 10.1161/CIRCRESAHA.116.308030

25. Farhangi MA, Najafi M, Asghari Jafarabadi M, Jahangiry L. Mediterranean dietary quality index and dietary phytochemical index among patients candidate for coronary artery bypass grafting (CABG) surgery.
BMC Cardiovasc Disord. (2017) 17:114. doi: 10.1186/s12872-0170544-z

26. Mirmiran P, Esfahani FH, Mehrabi Y, Hedayati M, Azizi F. Reliability and relative validity of an FFQ for nutrients in the Tehran lipid and glucose study. Public Health Nutr. (2010) 13:654-62. doi: 10.1017/S1368980009991698

27. Moludi J, Alizadeh M, Davari M, Golmohammadi A, Maleki V. The efficacy and safety of probiotics intervention in attenuating cardiac remodeling following myocardial infraction: Literature review and study protocol for a randomized, double-blinded, placebo controlled trial. Contemp Clin Trials Commun. (2019) 15:100364. doi: 10.1016/j.conctc.2019.100364

28. Anand IS, Latini R, Florea VG, Kuskowski MA, Rector T, Masson S, et al. C-reactive protein in heart failure: prognostic value and the effect of valsartan. Circulation. (2005) 112:1428-34. doi: 10.1161/CIRCULATIONAHA.104.508465

29. Rauchhaus M, Doehner W, Francis DP, Davos C, Kemp M, Liebenthal C, et al. Plasma cytokine parameters and mortality in patients with chronic heart failure. Circulation. (2000) 102:3060-7. doi: 10.1161/01.CIR.102.25.3060

30. Anker SD, von Haehling S. Inflammatory mediators in chronic heart failure: an overview. Heart. (2004) 90:464-70. doi: 10.1136/hrt.2002.007005

31. Basu A, Devaraj S, Jialal I. Dietary factors that promote or retard inflammation. Arterioscler Thrombo Vasc Biol. (2006) 26:995-1001. doi: 10.1161/01.ATV.0000214295.86079.d1

32. Neufcourt L, Assmann KE, Fezeu LK, Touvier M, Graffouillère L, Shivappa $\mathrm{N}$, et al. Prospective association between the dietary inflammatory index and cardiovascular diseases in the supplémentation en vitamines et minéraux antioxydants (SU. VI. MAX) cohort. J Am Heart Assoc. (2016) 5:e002735. doi: 10.1161/JAHA.115.002735

33. Holt EM, Steffen LM, Moran A, Basu S, Steinberger J, Ross JA, et al. Fruit and vegetable consumption and its relation to markers of inflammation and oxidative stress in adolescents. J Am Dietetic Assoc. (2009) 109:414-21. doi: 10.1016/j.jada.2008.11.036

34. Raff M, Tholstrup T, Basu S, Nonboe P, Sørensen MT, Straarup EM. A diet rich in conjugated linoleic acid and butter increases lipid peroxidation but does not affect atherosclerotic, inflammatory, or diabetic risk markers in healthy young men. J Nutr. (2008) 138:509-14. doi: 10.1093/jn/138.3.509

35. Galland L. Diet and inflammation. Nutr Clin Pract. (2010) 25:634-40. doi: $10.1177 / 0884533610385703$

36. Ruiz-Canela M, Bes-Rastrollo M, Martínez-González MA. The role of dietary inflammatory index in cardiovascular disease, metabolic syndrome and mortality. Int J Mol Sci. (2016) 17:1265. doi: 10.3390/ijms17081265

37. de Souza RJ, Mente A, Maroleanu A, Cozma AI, Ha V, Kishibe T, et al. Intake of saturated and trans unsaturated fatty acids and risk of all cause mortality, cardiovascular disease, and type 2 diabetes: systematic review and meta-analysis of observational studies. BMJ. (2015) 351:h3978. doi: 10.1136/bmj.h3978

38. Dhibi M, Brahmi F, Mnari A, Houas Z, Chargui I, Bchir L, et al. The intake of high fat diet with different trans fatty acid levels differentially induces oxidative stress and non alcoholic fatty liver disease (NAFLD) in rats. Nutr Metabo. (2011) 8:65. doi: 10.1186/1743-7075-8-65

39. Garcia-Arellano A, Martínez-González MA, Ramallal R, Salas-Salvadó J, Hébert JR, Corella D, et al. Dietary inflammatory index and allcause mortality in large cohorts: the SUN and PREDIMED studies. Clin Nutr. (2019) 38:122131. doi: $10.1016 /$ j.clnu.2018.05.003

40. Corella D, Shivappa N, Schröder H, Hébert JR, Ros E, Fitó M, et al. Dietary Inflammatory Index and Incidence of Cardiovascular Disease in the PREDIMED Study.

41. Wirth MD, Shivappa N, Hurley TG, Hébert JR. Association between previously diagnosed circulatory conditions and a dietary inflammatory index. Nutr Res. (2016) 36:227-33. doi: 10.1016/j.nutres.2015.11.016

42. Freitas H, Barbosa EA, Rosa FH, Lima AC, Mansur AJ. Association of HDL cholesterol and triglycerides with mortality in patients with heart failure. Brazil J Med Biol Res. (2009) 42:420-5. doi: 10.1590/S0100-879X2009000500004

43. Rauchhaus M, Clark AL, Doehner W, Davos C, Bolger A, Sharma $\mathrm{R}$, et al. The relationship between cholesterol and survival in patients with chronic heart failure. J Am College Cardiol. (2003) 42:1933-40. doi: 10.1016/j.jacc.2003.07.016

44. Säemann MD, Poglitsch M, Kopecky C, Haidinger M, Hörl WH, Weichhart T. The versatility of HDL: a crucial anti-inflammatory regulator. Eur J Clin Investig. (2010) 40:1131-43. doi: 10.1111/j.1365-2362.2010.02361.x 
45. Michaud K, Augsburger M, Donzé N, Sabatasso S, Faouzi M, Bollmann $M$, et al. Evaluation of postmortem measurement of NT-proBNP as a marker for cardiac function. Int. J Legal Med. (2008) 122:415-20. doi: 10.1007/s00414-0080257-z

Conflict of Interest: JH owns controlling interest in Connecting Health Innovations LLC (CHI), a company that has licensed the right to his invention of the dietary inflammatory index (DII) from the University of South Carolina in order to develop computer and smart phone applications for patient counseling and dietary intervention in clinical settings. NS was an employee of CHI.
The remaining authors declare that the research was conducted in the absence of any commercial or financial relationships that could be construed as a potential conflict of interest.

Copyright (C) 2021 Moludi, Shivappa, Alisgharzadeh, Hébert and Alizadeh. This is an open-access article distributed under the terms of the Creative Commons Attribution License (CC BY). The use, distribution or reproduction in other forums is permitted, provided the original author(s) and the copyright owner(s) are credited and that the original publication in this journal is cited, in accordance with accepted academic practice. No use, distribution or reproduction is permitted which does not comply with these terms. 